\title{
Analysis of 176 cases of adrenal incidentaloma investigated in a single clinical center, What are we doing?
}

Maíz Jimenez, M.1; Calatayud Gutierrez, M. 1; Rodriguez Niño,V.2; Librizzi, S. 1; Partida, M1.; Hawkins Caranza, F. 1; Endocrinology and Nutrition Service. Hospital 12 de Octubre 1. Complutense University2. Madrid. Spain.

\section{INTRODUCTION:}

The number of patients referred to an endocrinologist for assesment of incidental adrenal mass is increasing. In most cases are bening lesions, non-functional and remain stable over time. Current clinical manegement is variable due to the lack of evidence, wich leads in some cases to unnecessary tests and follow up.

\section{OBJECTIVES:}

Analyze clinical, radiologic and hormonal characteristics of patients studied for adrenal incidentaloma (AI) in our service. Evaluate the number and utility of complementary tests solicited for its diagnosis, what outcome and how long were they followed up

\section{MATERIAL AND METHODS:}

Observational, retrospective study in patients evaluated for Al in the endocrinology service of a tertiary hospital from 1993-2014. We excluded those with symptoms of hormonal hyperproduction and those with insufficient data.

\section{RESULTS}

176 patients (111 women and 65 men) met inclusion criteria. The medium age was 59,68 $\pm 12,54$ years $(31-84)$.

Radiologjic study:

Each patient had a medium of $4.14 \pm 2,69$ radiologic tests (1-14), mainly CT and a medium follow up of $3,65 \pm 3,75$ years $(0-18)$

$64 \%$ of tumors measured less than $3 \mathrm{~cm}, 20 \%$ measured $3-4 \mathrm{~cm}$ and $16 \%$ measured more than $4 \mathrm{~cm}$ (Figure 1).

$29 \%$ of patients had bilateral disease (Figure 2 ).

With the first radiologyc test, $87 \%$ of the lesions were well characterized. The radiologyc diagnose was: adenoma (69\%), Hyperplasia (6\%), Mielolipoma (3\%), Suspicious of malignancy $(2 \%)$ and Pheochromocytoma $(1 \%)$

Only $13 \%$ (22) of them remained indetermined with the fist radiological test. This lesions, once completed the study, the final diagnose was: 14 adenomas (66\%), 5 Pheochromocytomas (24\%), 2 metastases (9\%) and ne still remained indetermined because the patient refused to continue the study.

\section{Funcional study:}

The hormonal test most frecuently solicited was the $1 \mathrm{mg}$ dexametasone suppression test (1mg DST) ACTH, urinary 24 hour cortisol and urinary cathecolamines. Medium laboratory follow up was

$3.75 \pm 3,5$ years $(0-17) .75 \%$ of patients had at least one laboratory test out of normal range at diagnose or during follow up, $1 \mathrm{mg}$ DST was the test most frequently altered in 80 patients $(47.3 \%)$ but only 33 patients $(18,7 \%)$ fulfilled criteria for subclinical Cushing syndrome (SCS)

After complete study (excluding the metastases) the functional diagnosis was: 64\% (107) Nonfunctioning adenoma; 19\% (32) Subclinical Cushing Syndrome, 4\% (8) Pheochromocytomas, 3\% (5) Clinical adrenal Cushing, 1 ACTH dependent Cushing. (Figure 4).

\section{Malignancy}

Metastases were diagnosed in 6 patients, all of them were detected in study of extension in patients with known neoplasia. No adrenal carcinoma was diagnosed.

\section{Outcomes:}

The majority of patients remained stable. 7 patients that were initially diagnosed of non-functioning adenoma, developed SCS. No patients with SCS developed clinical Cushing (Figure 5)

Only 4 tumors growed significantly, all of them were metastases (Figure 6).

20 Patients required surgery: 5 adrenal Cushing, 6 SCS, 8 pheochromocytomas and 1 metastases. None of the lesions that were considered bening at diagnosis, suffered from malignant transformation to adrenal carcinoma. One patient with colon carcinoma and initial diagnose of adenoma was diagnosed of metastasis after 6 years of follow up and died because of malignant disease.

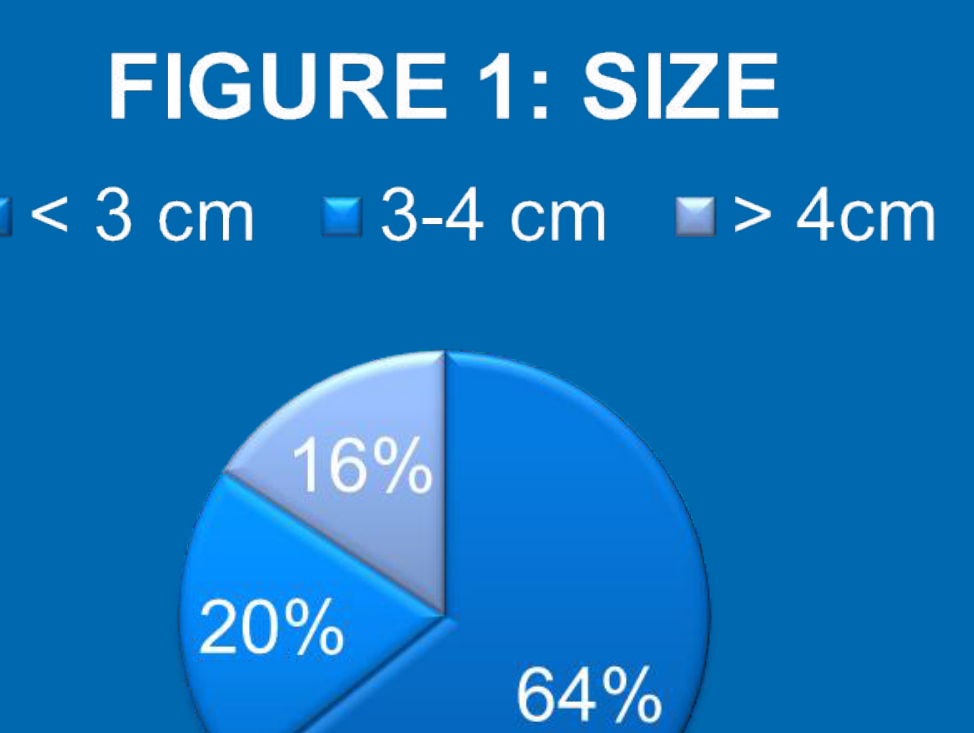

FIGURE 2

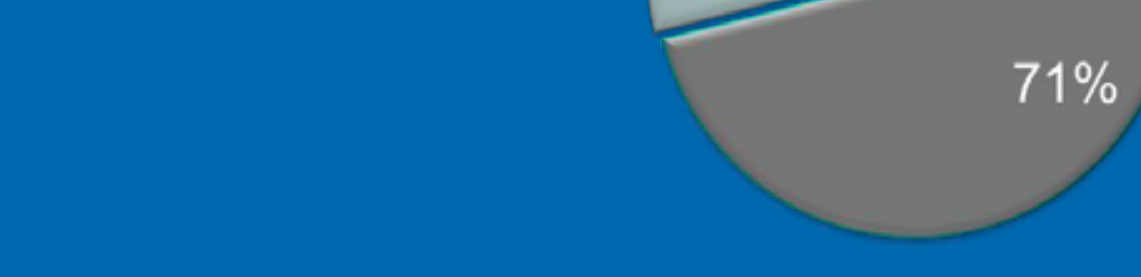

FIGURE 3:

RESULTS OF THE FIRST RADIOLOGYC TEST

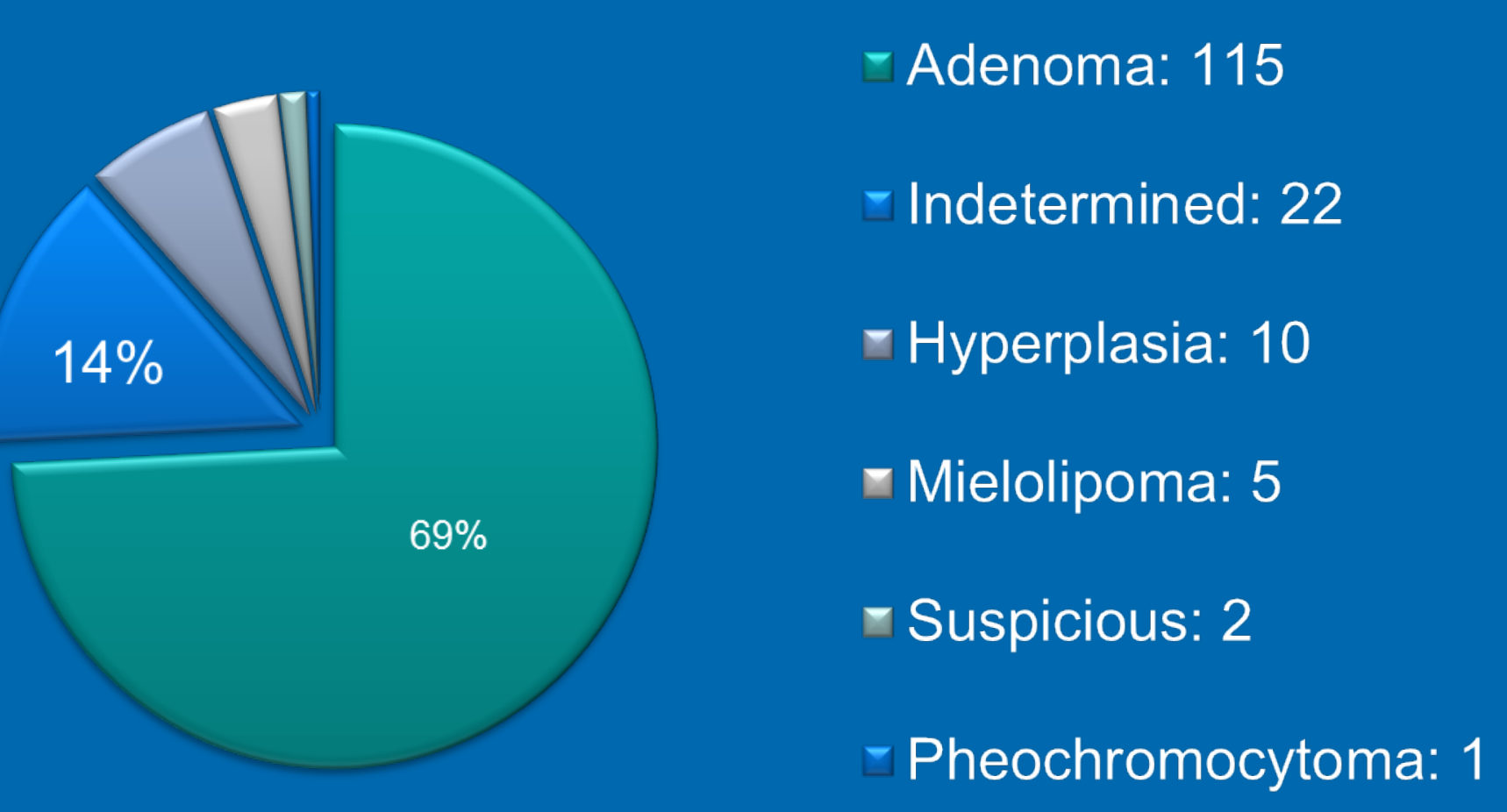

FIGURE 4: FUNCTIONAL DIAGNOSIS
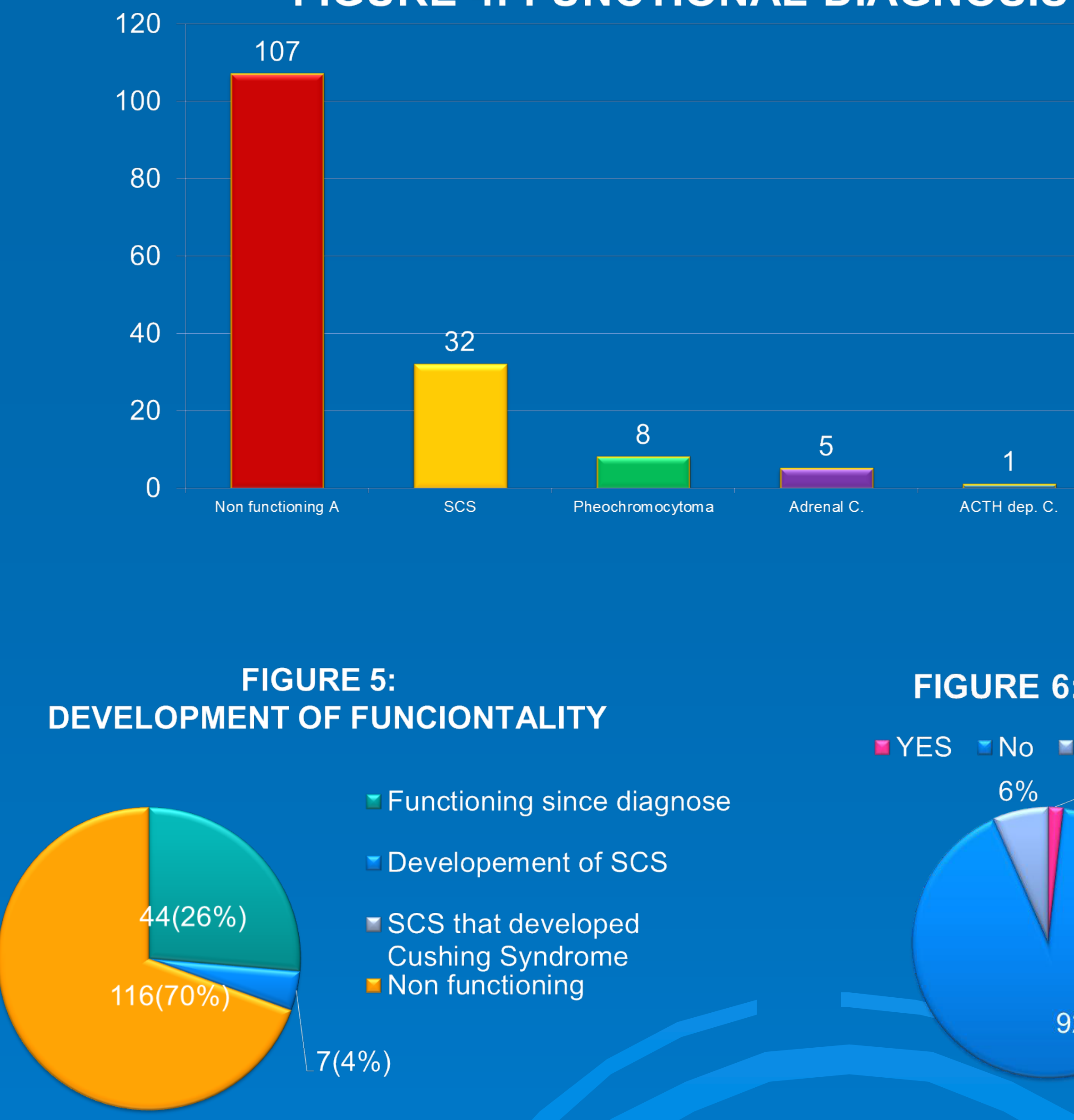

FIGURE 6: GROWTH

IYES -NO -UNKNOWN 